\title{
Principales Retos en el Tratamiento Grupal de los Hombres Condenados por un Delito de Violencia de Género
}

\section{Main Challenges in Group Treatment for Men Convicted of a Crime of Gender Violence}

\author{
Santiago Boira \\ Universidad de Zaragoza, España \\ Pablo Carbajosa \\ Instituciones Penitenciarias, SGPMA de Almería, España \\ Marisol Lila \\ Universidad de Valencia, España
}

\begin{abstract}
Resumen. El objetivo de este artículo es analizar algunos aspectos importantes a considerar en la intervención grupal que se realiza en los programas psico-educativos dirigidos a los hombres condenados por un delito de violencia de género en España. Para ello, en primer lugar, se repasan los temas relacionados con la violencia de género que implican importantes consecuencias para el diseño e implementación de los programas de tratamiento dirigidos a los hombres. En segundo lugar, se identifican las circunstancias y dificultades relacionadas con el contexto del tratamiento, el penado y el terapeuta que pueden ser determinantes en la dinámica grupal y el desarrollo del tratamiento. Finalmente, se identifican algunos retos que debe enfrentar el terapeuta en este tipo de intervenciones.

Palabras clave: violencia contra la pareja, tratamiento grupal, intervención psicológica, programas para maltratadores, violencia de género.
\end{abstract}

\begin{abstract}
The aim of this paper is to analyze some important aspects to consider in the group intervention carried out in the psycho-educational programs for men convicted of a crime of gender violence in Spain. To do this, first, we study the issues related to gender violence involving important consequences for the design and implementation of treatment programs for men. Second, we identify the factors and difficulties related to the context of treatment, the offender and the therapist which may be decisive in group dynamics and development of treatment. Finally, some challenges facing the therapist in such interventions are specified.

Keywords: intimate partner violence, group treatment, psychological intervention, batterer programs, gender violence.
\end{abstract}

\footnotetext{
La correspondencia sobre este artículo debe enviarse a Santiago Boira. Facultad de Ciencias Sociales y del Trabajo. Universidad de Zaragoza. C/ Violante de Hungría, 23. 50009-Zaragoza (España). E- mail: sboira@unizar.es
} 


\section{Las dificultades en el tratamiento de los agresores de género}

La erradicación de la violencia dentro de la pareja sigue siendo un problema grave y un reto que implica al conjunto de la sociedad. En este sentido, la Organización Mundial de la Salud, en el informe realizado en 2013, señala que se trata de un problema de proporciones epidémicas. En España es fundamentalmente a partir de la entrada en vigor de la Ley 1/2004 de Medidas de Protección Integral contra la Violencia de Género, cuando se intensifican las acciones de lucha contra este tipo de violencia. Las estrategias de actuación se han centrado en visibilizar el problema, en mejorar los medios para la atención de las víctimas y en el endurecimiento de las actuaciones contra los hombres violentos. En los últimos años ha aumentado de manera significativa tanto el número de agresores que han ingresado en prisión como el número de condenados obligados a realizar un programa de tratamiento (Secretaría General de Instituciones Penitenciarias, 2011).

Los modelos que han tratado de explicar la violencia masculina dentro de la pareja se podrían agrupar en cuatro grandes categorías: a) aquellos que acentúan la causa de la violencia en variables intrapersonales, b) los que se centran en variables interpersonales, c) los que colocan la causa de la violencia fundamentalmente en variables de carácter sociocultural, y d) los que analizan la violencia como una interrelación de los tres anteriores (modelo ecológico).

Respecto a su definición, este tipo de violencia puede entenderse como un intento sistemático por parte del agresor de conseguir y mantener el control de la relación utilizando un comportamiento violento en sus distintas manifestaciones. Para conseguir este objetivo, la utilización de una u otra modalidad de violencia no suele ser lo más relevante y las características de este comportamiento, su grado de brutalidad, depende de la propia historia de aprendizaje del hombre respecto al uso de la violencia, la tensión en el momento del incidente o de la evaluación cognitiva sobre los comportamientos de su mujer. De hecho, la elección de un tipo u otro de comportamiento (incluidos los comportamientos positivos y de seducción) suele supeditarse al mantenimiento de esta posición ventajosa (Boira, 2010).

Habitualmente, el fin último del comportamiento violento no suele ser el daño sino el control. Tanto en el caso del daño psicológico como en el caso de la agresión física o sexual, el hombre suele presentarlo como algo accidental utilizando mecanismos de negación, minimización y externalización de la responsabilidad que dificultan la conciencia y mantienen el comportamiento (Lila, Gracia y Herrero, 2012). De este modo, la percepción que el agresor tiene de su comportamiento se caracteriza por la ausencia de conciencia de problema, lo que incide en una comprensión muy distorsionada de la situación y de una deficiente capacidad empática para ponerse en el lugar de la mujer (Romero-Martínez, Lila, Catalá-Miñana, Williams y Moya-Albiol, 2013). Dicha cuestión se convierte en uno de los primeros y principales escollos en el proceso de rehabilitación. En este sentido, el reconocimiento y la empatía deberían constituirse como elementos fundamentales de los programas de actuación que pueden incidir directamente en la protección y prevención del comportamiento violento.

Asimismo, un aspecto muy importante que debe tenerse en cuenta en los procesos educativos y de rehabilitación es el modo en que se van fraguando las actitudes y los valores que desembocan en un comportamiento violento y que, como se ha visto, no necesariamente involucran violencia física. Diferentes investigaciones ponen de manifiesto la cada vez más temprana edad en la que aparecen actitudes sexistas sobre el modo de entender el amor o el papel del hombre y la mujer dentro de una relación (Lemus y Ryan, 2010; Diaz-Aguado, 2006; Rodríguez, Antuña, López-Cepero, Rodríguez, Herrero y Bringas, 2008) o como existen y se fomentan en determinados colectivos actitudes sociales que favorecen y sustentan la violencia contra la mujer en las relaciones de pareja (Gracia, García y Lila, 2009; Gracia, Herrero, Lila y Fuente, 2009).

En relación con los tratamientos, las revisiones internacionales que han evaluado los programas han mostrado resultados discretos. También en España han ido publicándose en estos últimos años estudios en los que se examina la efectividad de este tipo de intervenciones (e.g., Boira, López, Tomás-Aragonés y Gaspar, 2013; Echeburúa, Sarasua, Zubizarreta y De Corral, 2009; Lila, Oliver, Galiana y Gracia, 2013; Novo, Fariña, Seijo 
y Arce, 2012; Pérez, Giménez-Salinas y de Juan, 2013). El debate acerca de si la evidencia indica que estos programas tienen éxito o no continua y se ha convertido en una cuestión controvertida (Bowen, 2011; Eckhardt et al., 2013; Feder, Wilson y Austin, 2008; Lila, 2013), siendo un importante desafío identificar los aspectos que puedan mejorar su eficacia (Saunders, 2008). En este sentido, la mejora de la seguridad de la víctima, la prevención de los posibles daños futuros, el aumento de la conciencia sobre el daño cometido y el cambio del sistema de creencias se convierten en elementos críticos de la intervención.

En España, este tipo de programas se aplican bajo tres condiciones: a) mediante la participación voluntaria del hombre; b) como consecuencia de una medida judicial que sustituye o suspende una pena privativa de libertad; y c) una vez que el hombre se encuentra en prisión. Debido al aumento considerable de hombres condenados por este tipo de violencia, la modalidad obligatoria es la más ejecutada y la que plantea mayores retos técnicos que pueden complicar sobremanera la obtención de resultados positivos. Millana (2011) recoge las características de las principales propuestas terapéuticas que actualmente se realizan en España dirigidas a hombres condenados por violencia de género (Arce y Fariña, 2007; Echauri, Romero y Rodríguez, 2005; Graña, Muñoz, Redondo y González, 2008; Lila, García y Lorenzo, 2010; Quinteros y Carbajosa, 2008; Ruiz y Expósito, 2008).

En este contexto, otro aspecto importante a considerar es la elección del tipo de formato de tratamiento: individual o grupal (Boira, López et al., 2013). El tratamiento de elección suele tener mayoritariamente un formato grupal (Price y Rosembaum, 2009). Sin embargo, hay que tener presente las posibilidades de las intervenciones individuales que, entre otras, presentan la ventaja de una mayor adaptación a las características específicas de los penados (Murphy y Eckhardt, 2005).

En el panorama internacional, alguna de las cuestiones objeto de debate en relación con la intervención grupal se ha centrado en determinar el enfoque de tratamiento considerado como más adecuado. Respecto a la eficacia, dicho debate se basa principalmente en la opción por un tipo de intervención basada en los factores de riesgo frente a otro centrado en las fortalezas o aspectos positivos de los agresores (e.g., Bouman, Schene y Ruiter, 2009; Langlands, Ward y Gilchrist, 2009; Lila, Gracia y Murgui, 2013). A nivel práctico esta dualidad se traduce, entre otras cosas, en orientar el programa desde un enfoque psicoterapéutico centrado en el cliente e individualizado frente a un tratamiento de carácter psicoeducativo, generalista y con un mayor grado de estructuración. Andrews, Bonta y Wormith (2011) señalan que este debate es actualmente vigoroso, planteado globalmente en los entornos penitenciarios y, asimismo, trasladado al ámbito de la intervención grupal con agresores de pareja (Gondolf, 2012). No obstante, a día de hoy, tanto en el contexto internacional como en nuestro país, la modalidad de tratamiento mayoritariamente elegida es de tipo psicoeducativa altamente estructurada (Carbajosa y Boira, 2013; Price y Rosembaum, 2009).

Por otra parte, la intervención con esta población fundamentalmente en los programas por mandato judicial plantea muchos problemas debido a la dificultad para que los hombres asuman su comportamiento y a su baja motivación (Murphy y Baxter, 1997; Quinteros y Carbajosa, 2008; Scott, King, McGinn y Hosseini, 2011). Al desarrollarse en un contexto coercitivo y de obligatoriedad la intervención se diferencia en algunos aspectos esenciales de un planteamiento terapéutico más convencional. En este caso, una de las primeras tareas será la búsqueda de activadores y movilizadores del cambio (Cirillo, 1994). Así, elementos como la motivación, la alianza terapéutica, las habilidades del terapeuta o el contexto concreto en el que el programa se desarrolla pueden intervenir decididamente en la eficacia del tratamiento (Boira, Del Castillo, Carbajosa y Marcuello, 2013; Carbajosa y Boira, 2013; Carbajosa, Boira y Tomás-Aragonés, 2013; Lila, Gracia et al., 2013; Lila, Oliver, Galiana et al., 2013).

De hecho, el modo en que se manejen estas cuestiones por parte de los facilitadores del grupo puede resultar esencial en la obtención de resultados positivos (Bowen, 2011). Es más, la interrelación entre los distintos factores macro y micro de la intervención no pueden analizarse de manera aislada, siendo necesaria una visión ecológica que contemple las relaciones de influencia entre contexto, terapeuta, programa y penados (Andrews et al., 2011; Edleson y Tolman, 1992; Silvergleid y Mankowski, 2006). 
Este artículo analiza algunos aspectos importantes a considerar en la intervención grupal que se realiza en los programas psico-educativos dirigidos a los hombres condenados por un delito de violencia de género en España. No obstante, aunque este trabajo se centra en el proceso grupal, no pueden dejarse al margen factores como la obligatoriedad del tratamiento o el contexto institucional desde donde se implementan los programas, los cuales van a condicionar los objetivos de la intervención, las actitudes de los participantes y la necesidad de desarrollar habilidades específicas por parte del terapeuta.

\section{El contexto de la intervención: obligatoriedad y alianza terapéutica}

Como se ha comentado, la primera dificultad que encuentra el terapeuta a la hora de intervenir con hombres obligados a realizar un programa de reeducación es el propio contexto donde se enmarca la terapia. En estos casos, la intervención viene caracterizada por una limitación de la capacidad de maniobra del terapeuta condicionada por: a) el contexto judicial; b) el programa que debe ser aplicado; y c) el enfoque del tratamiento.

Una vez que se ha considerado la violencia dentro de la pareja como crimen y se ha condenado a su responsable, es necesario establecer un marco de tratamiento que ofrezca al maltratador la oportunidad de modificar su comportamiento (Lila, Gracia et al., 2013). Sin embargo, esta situación conforma un escenario de tratamiento muy distinto al habitual en terapia que se podría caracterizar por una mayor motivación para recibir apoyo y una relación de colaboración con el terapeuta (Ross, Polaschek y Ward, 2008).

Las condiciones iniciales en un medio coercitivo son muy diferentes a las que se producen en otros contextos de terapia (e.g., imposición del programa al penado o el requerimiento al profesional de informar al juzgado), lo que puede dificultar el establecimiento de una adecuada alianza terapéutica (Kozar y Day, 2012; Ross et al., 2008). Este hecho puede resultar fundamental para evitar abandonos e influir en la reducción futura del comportamiento violento (Boira, López et al., 2013; Lila, Oliver, Galiana et al., 2013; Taft, Murphy, Musser y Remington 2004; Taft y Murphy, 2007).

Sin embargo, la identificación de los terapeutas como parte del sistema coercitivo es inevitable. Para un adecuado progreso es fundamental afrontar este aspecto desde el principio, siendo muy claro en el enfoque de la intervención al tiempo que se mantiene una actitud empática. El profesional debe ser capaz de separar el rechazo hacia la conducta violenta por la que estos hombres han sido condenados, de su cualidad de seres humanos capaces de cambiar y mejorar su forma de relación con los demás. El objetivo es transmitir al penado la posibilidad de compaginar el contexto coercitivo con el establecimiento de una relación de ayuda que le aporte beneficios y mejore su situación personal (Sonkin y Dutton, 2003). En un contexto coercitivo la creación y mantenimiento de alianza y la reparación de las rupturas de la misma requiere un amplio repertorio de habilidades y un alto nivel de competencia del terapeuta (Kozar y Day, 2012). En este sentido, algunas estrategias deben orientarse a: a) ofrecer información útil para el penado tratando de solventar las dudas sobre el proceso penal y evitando activar sus mecanismos de defensa (Miller y Rollnick, 2002); b) conectar con el proceso emocional que vive el penado habitualmente asociado con sentimientos de rabia, impotencia, vergüenza y miedo por la pérdida de la pareja y de los hijos. El impacto emocional, a menudo incrementado en el caso de los juicios rápidos, provoca confusión en el agresor en un contexto en el que a menudo desconoce la dinámica judicial y sus consecuencias. La atención genuina, la posibilidad para los hombres de expresar dentro del grupo sus sentimientos y la labor de contención y de información del terapeuta ayuda a generar un vínculo de confianza. En este sentido, según Ackerman y Hilsenroth (2003), la habilidad para entender y conectar con la experiencia del paciente resultará un componente fundamental para la construcción de la alianza. Además, si el penado percibe que el terapeuta es competente aumentará su motivación y la posibilidad de establecer una buena alianza terapéutica. En definitiva, debe creer y sentir que el terapeuta puede ayudarle para poder avanzar en el proceso terapéutico (Taft y Murphy, 2007). 


\section{El penado como sujeto de tratamiento}

Junto con las dificultades derivadas del contexto se producen otras derivadas de la problemática de los propios agresores, que también suponen un importante reto para el terapeuta y para la dinámica que en el grupo se va a desarrollar. En este apartado se van a comentar las referidas a la motivación, la conciencia de problema por parte del penado, el ajuste psicosocial del penado y las actitudes hacia la terapia y el terapeuta.

\section{Motivación}

La primera de ellas es la baja motivación inicial con la que el penado acude al tratamiento como consecuencia, en parte, de la obligación judicial de realizarlo y evitar así el ingreso en prisión (Taft et al., 2004). En este contexto, el participante en el programa no se incorpora con una motivación interna y genuina de resolver su problema (Kristenmacher y Weiss, 2008). Además, su predisposición al cambio no es uniforme y el hecho de participar en el programa no garantiza que se comience a cambiar (Eckhardt, Babcock y Homack, 2004). Al abordar este tema es importante que el terapeuta tenga en cuenta que la falta de motivación inicial no es determinante y podrá variar a lo largo del proceso terapéutico, incluso en los casos más resistentes (Polaschek y Ross, 2010).

Así, centrar la intervención sobre la motivación hacia el cambio debe ser un objetivo prioritario, tanto al inicio como durante todo el tratamiento (Redondo, 2010). Para intervenir sobre la falta de motivación, diversos autores proponen la utilización del modelo transteórico de los procesos de cambio (Prochaska y Diclemente, 1986) para abordar la comprensión del proceso de preparación para el cambio en maltratadores y ajustar las intervenciones y programas a dicho proceso (Alexander, Morris, Tracy y Frye, 2010; Eckhardt et al., 2004; Eckhardt, Samper y Murphy, 2008; Quinteros y Carbajosa, 2008; Scott y Wolfe, 2003; Scott., 2004; Zalmanowitz, Babins-Wagner, Rodger, Corbett y Leschied, 2013).

Además de tener en cuenta los distintos estadios del proceso de cambio y sus fluctuaciones, en este tipo de programas un elemento clave en la intervención es la aplicación de estrategias de retención proactiva y motivacionales específicas que fomenten el aumento de la motivación de los penados, su adherencia al tratamiento y disminuyan el riesgo de abandono (Echeburua, 2013; Lila, 2013; Lila, Gracia et al., 2013; Murphy, Linehan, Reyner, Musser y Taft, 2012; Subirana-Malaret y Andrés-Pueyo, 2013; Zalmanowitz et al., 2013). La combinación del conocimiento del proceso de cambio del penado y sus estadios y aplicar las técnicas motivacionales adecuadas para cada estadio puede resultar determinante en el resultado de la intervención (Murphy et al., 2012; Zalmanowitz et al., 2013).

\section{Conciencia del problema}

La negación de la violencia o la minimización de su gravedad representan un comportamiento habitual en un grupo significativo de maltratadores (Lila et al., 2012; Lila, Herrero y Gracia, 2008). El penado no identifica esta conducta como delito ni valora el impacto que supone para la víctima. Asimismo, habitualmente se externaliza la responsabilidad culpando al sistema judicial o a la víctima de su situación actual (Boira, Del Castillo et al., 2013; Henning, Jones y Holdford, 2005; Lila, Oliver, Catalá-Miñana, Galiana y Gracia, 2014; Quinteros y Carbajosa, 2008). El agresor desarrolla un conjunto de mecanismos de defensa que se traducen en una resistencia inicial al tratamiento o rechazo de la terapia.

En el análisis que realizaron Marshall et al. (2002) sobre las habilidades del terapeuta en la intervención con agresores sexuales, una de sus principales conclusiones fue que la actitud reforzadora y directiva de los terapeutas disminuía la atribución de la responsabilidad a la víctima. Y, en esta misma línea, una actitud empática 
y cálida del terapeuta disminuye la tendencia a la minimización y negación del problema. Parece, además, que puede resultar más efectivo para abordar los mecanismos de defensa un estilo firme que exprese apoyo y refuerce al penado pero que evite la confrontación directa sistemática (Marshall, 2005; Marshall et al., 2002). El estilo directivo debe fomentar la búsqueda autónoma del cliente de soluciones propias a sus problemas y el terapeuta debe reforzar con apoyo sólo cuando sea necesario. En este sentido se debe reforzar la consecución de pequeños cambios y su generalización fuera de la terapia.

Ante esta dificultad de reconocimiento del problema, algunos autores en el ámbito de estudio de los maltratadores destacan la importancia de establecer un vínculo adecuado con aquellos penados que externalizan su responsabilidad y se muestran resistentes a las intervenciones del terapeuta (Taft, Murphy, King, Musser y DeDeyn, 2003). En estos casos, donde lo habitual es la negación, se sugiere de nuevo la necesidad de evitar las confrontación en las etapas iniciales de la terapia para no interferir negativamente en el establecimiento de la alianza terapéutica (Taft y Murphy, 2007).

En definitiva, parece necesario que el terapeuta maneje un estilo flexible que haga posible la asunción de responsabilidad, el reconocimiento del problema y el afrontamiento de los mecanismos de defensa. Esta flexibilidad debe mantener un equilibrio entre el progresivo cuestionamiento de las distorsiones cognitivas sobre la mujer y el uso de la violencia y una actitud empática que refuerce los pequeños cambios que pueda ir dando el penado (Rasanen, Holma y Seikkula, 2012; Silvergleid y Mankowski, 2006).

\section{Ajuste Psicosocial}

Algunos estudios han señalado la existencia de una elevada prevalencia de sintomatología depresiva entre los hombres que se encuentran participando en programas de intervención para maltratadores (Graham, Bernards, Flynn, Tremblay y Wells, 2012; Novo et al., 2012), sugiriendo la necesidad de tratar dicha sintomatología con el fin de reducir el riesgo de reincidencia. Igualmente, algunos estudios indican que los maltratadores tienden a tener una imagen distorsionada de sí mismos (e.g., Dutton y Golant, 1997; Murphy, Stosny y Morrel, 2005). En este sentido, las intervenciones para mejorar el ajuste psicológico de los maltratadores podrían incluir estrategias encaminadas a desarrollar una autoimagen y una autoestima ajustadas a la realidad y adaptativas (Lee, Sebold y Ukel, 2003; Murphy et al., 2005; Redondo, Martínez-Catena y Andrés-Pueyo, 2012).

En un reciente estudio realizado por Lila, Gracia et al. (2013), se sugiere que, no sólo el ajuste psicológico debe ser un objetivo potencial en los programas de intervención con maltratadores, sino también sus determinantes psicosociales. Entre los determinantes del ajuste, estos autores destacan el apoyo social y los eventos vitales estresantes. El aislamiento social (o la ausencia de una red de apoyo social) y la acumulación de eventos vitales estresantes, que caracterizan a buena parte de los penados que acuden a los programas de intervención, se encuentran relacionados con el desajuste psicológico y la conducta violenta de manera que, no sólo incrementan la probabilidad de ocurrencia de la misma, sino también su continuidad en el tiempo (Gracia, Herrero et al., 2009; Lanier y Maume, 2009; Silver y Teasdale, 2005). El apoyo social puede ayudar a resolver los conflictos en las relaciones íntimas y funcionar como un factor protector. En este sentido, el apoyo social puede ayudar a afrontar de forma más adecuada los eventos vitales estresantes y proporcionar a la pareja los recursos necesarios para solucionar los conflictos (Silver y Teasdale, 2005). Sin embargo, a pesar del potencial de estas variables, exceptuando unos pocos estudios (e.g., Choi, Cheung y Cheung, 2012; Lila, Oliver, Lorenzo y Catalá, 2013; Lila, Gracia et al., 2013), no se encuentra en la literatura científica investigaciones que examinen el estrés y el apoyo social en los hombres condenados por violencia contra la mujer en las relaciones de pareja. Sería interesante explorar estrategias de intervención novedosas en este ámbito tales como, por ejemplo, promover la relación de los penados con miembros de su comunidad con actitudes no tolerantes con la violencia, o promover las relaciones de apoyo entre los participantes de un grupo de intervención. Evidentemente, hay que tomar precauciones al utilizar estas estrategias, en la medida que algunas investigaciones señalan que en el caso de los mal- 
tratadores es habitual encontrar entre sus redes de apoyo miembros que toleran o condonan la violencia en las relaciones de pareja (Agoff, Herrera y Castro, 2007; Choi et al., 2012; Lila, Oliver, Lorenzo et al., 2013). El profesional debe estar especialmente alerta ante este tipo de relaciones en la intervención grupal para minimizar su impacto. Por otra parte, en relación a los eventos vitales estresantes, es frecuente que entre los objetivos de la terapia se encuentre el entrenamiento en estrategias de afrontamiento, técnicas de control de estrés, restructuración cognitiva y técnicas de solución de problemas, así como otras técnicas tradicionalmente utilizadas en los acercamientos cognitivo-conductuales, que pueden ayudar a los penados a afrontar los eventos vitales estresantes y resolver los conflictos y problemas de una forma más adaptativa y exenta de violencia.

\section{Actitudes hacia la terapia y el terapeuta}

Como consecuencia de la falta de conciencia del problema y del contexto en el que el programa se desarrolla, es habitual que el terapeuta se encuentre al comenzar la terapia con actitudes hostiles y desvalorización de sus intervenciones por parte del participante en el programa. Como ya se ha indicado, el terapeuta representa la cara de un sistema judicial que considera injusto y culpable de la situación en la que se encuentra (Lila et al., 2014). Desde esta perspectiva, el agresor suele tratar inicialmente de mantener el poder en la relación terapéutica a través de manipulaciones que intentan culpabilizar al terapeuta de su situación. Ante una situación que no controla, la tendencia es descargar de forma hostil sobre el terapeuta la rabia e impotencia que sienten (Taft y Murphy, 2007).

No obstante, junto con este tipo de actitudes hostiles, es también frecuente la presencia de un alto nivel de deseabilidad social en algunos hombres (Eckhardt, Holtzworth- Munroe, Nolander, Sibley y Cahill, 2008; Henning et al., 2005). Si en un primer momento son habituales las descargas de rabia e ira, conforme se avanza en el tratamiento se van alternando estas reacciones de indignación ante lo que el percibe como una injusticia con la pretensión de mostrar la cara positiva de si mismos. Además, en una etapa avanzada del programa pueden producirse resistencias a reconocer el comportamiento violento por miedo al juicio de los demás o las posibles consecuencias judiciales que pudieran derivarse. Por ello, es habitual que el hombre trate de mostrar en el grupo una versión positiva de su predisposición al cambio para adaptarse al contexto y evitar posibles informes negativos al juzgado. Para detectar el comportamiento real de los agresores en su entorno y compensar la deseabilidad social, parece imprescindible el acceso a fuentes de información colaterales de los distintos organismos ocupados del seguimiento de estos casos (Policía, Servicio de atención y protección de víctimas, organismos judiciales), así como los datos recogidos en la sentencia (Henning et al., 2005; Quinteros y Carbajosa 2008).

Finalmente, un aspecto a tener en cuenta por parte del terapeuta para la creación y fomento de una buena cohesión grupal son los aspectos que los penados valoran positivamente del programa. Entre ellos, destaca el ambiente de apoyo generado en el grupo. Respecto a los diferentes contenidos que se incluyen en estos programas se valoran los relacionados con la responsabilidad sobre su comportamiento, la motivación para cambiar, el control de la ira y autocontrol, así como las estrategias para poder evitar la violencia en el futuro (Boira, López et al., 2013).

\section{A modo de conclusión}

$\mathrm{Si}$, en términos generales, el terapeuta que interviene con hombres con problemas de control y violencia en el hogar debe enfrentarse a situaciones complejas, cuando la intervención se realiza en un contexto grupal y con hombres condenados a realizar el programa las dificultades se intensifican. El futuro de este tipo de programas pasa por encontrar un equilibrio en atender a los factores de riesgo (demanda institucional y social) sin perder 
de vista el bienestar del penado (demanda terapéutica). El atender estos dos factores de manera conjunta y efectiva es quizás el mayor desafío de este tipo de programas y, para ello, hay que cumplir los objetivos y contenidos del programa, primando en todo momento la seguridad de la víctima. Por otro lado, desde una visión psicoterapéutica, hay que atender a las necesidades de cada individuo y considerar desde un interés clínico aspectos como la alianza, motivación o características individuales de los penados (Stewart, Flight y Slavin-Stewart, 2013). Este tema es actualmente una cuestión de intenso debate en las intervenciones desde el sistema judicial y en el campo específico de los agresores de género, siendo un punto clave que diferencia este tipo de intervención de las que se producen en otros ámbitos.

Debido al contexto institucional en el que se implementan estos programas, el primer reto con el que se encuentra el terapeuta en este tipo de grupos de carácter psicoeducativo es el riesgo de caer en la excesiva "manualización". El cumplimiento de dichas demandas institucionales, la atención a los contenidos de los programas que mantenga la integridad de los tratamientos y la permanente alerta ante los factores de riesgo, pueden dificultar la conexión con las vivencias personales de los participantes del grupo y con sus necesidades terapéuticas individuales (Andrews et al., 2011). En este caso, sin perder de vista los objetivos y tareas propuestos por el programa, es importante para generar una buena alianza de trabajo establecer acuerdos en las tareas y objetivos a conseguir entre el terapeuta y el grupo (Bordín, 1979).

En la intervención grupal, en relación a las habituales reacciones de ira de los penados al comienzo del programa, el terapeuta debe poseer capacidad empática para no personalizar los ataques ni cuestionarse su intervención. En este sentido, debe ofrecer un rol alternativo de apoyo, que trate de desmontar las creencias del penado habitualmente basadas en que las relaciones interpersonales requieren de la coerción y el control (Taft et al., 2003). Además, el terapeuta debe ser asertivo para manejar la agresividad dirigida hacia él y poner límites a este tipo de situaciones. Finalmente, también será necesaria una actitud segura que mantenga y facilite el encuentro terapéutico (Quinteros y Carbajosa, 2008). Para el manejo de pacientes hostiles y altamente resistentes es necesario aplicar un proceso previo motivacional que se caracterice por una actitud empática, que evite la confrontación y refuerce los cambios positivos y la predisposición para trabajar los posteriores contenidos del programa (Scott et al., 2011).

Por otro lado, el terapeuta debe ser capaz de modular su estilo terapéutico en función del avance del grupo. Probablemente al comienzo de la intervención se requiera de un estilo más directivo y participativo para establecer la dinámica grupal, clarificar el contexto de trabajo y establecer las reglas de la intervención. Con posterioridad, una vez que los penados asumen la situación de obligatoriedad y comienzan a ver los beneficios de participar en el grupo, esta situación puede y debe cambiar. A partir de este momento, el terapeuta puede tener la posibilidad de actuar como agente facilitador del cambio sin que sean necesarios unos límites tan estrictos y en donde cabe esperar que los penados participen desde su propia motivación (Quinteros y Carbajosa, 2008).

El mantener estas demandas equilibradas es quizás uno de los mayores desafíos de este tipo de grupos. Para ello la flexibilidad del terapeuta debe encontrar un adecuado balance entre el cuestionamiento y apoyo y detectar cuándo es necesaria una mayor estructuración a través de los contenidos del programa y cuándo se debe enfocar la intervención en un estilo menos estructurado centrado en las necesidades individuales de alguno de los participantes (Rasanen et al., 2012). Asimismo, debe ser capaz de generar una adecuada cohesión grupal -aspecto muy valorado por los penados- en la que el participante perciba tanto el apoyo por parte del grupo como del terapeuta (Rosenberg, 2003; Taft y Murphy, 2007).

Finalmente, para concluir queremos hacer referencia al terapeuta como sujeto. Este asunto ha recibido poca atención en comparación con el otorgado a las características de los programas aplicados y, sin embargo, puede resultar esencial en este contexto (Carbajosa et al., 2013). La primera dificultad que debe gestionar el terapeuta en el contexto coercitivo es la definición de su rol, el cual va a alternar entre una versión orientada hacia la ayuda y otra hacia el control. Dentro de este contexto y, como ya se ha señalado, el terapeuta debe ir compaginando dos roles de manera continua. Por un lado, como agente de ayuda del penado y, por otro, agente perteneciente al sistema judicial, que debe informar y controlar los avances de los penados. Esta duplicidad de roles 
suele generar conflictos y vivencias contradictorias en el terapeuta, al tener que informar y tomar decisiones que pueden tener consecuencias para el penado (como en el caso de comunicaciones del incumplimiento de una orden de alejamiento o al emitir informes negativos que pueden suponer el ingreso en prisión de los penados) (Ross et al., 2008).

Asimismo, el terapeuta va a encontrarse con las resistencias de los participantes en el grupo, su negación de los hechos, la ausencia de avance terapéutico o con actitudes de hostilidad hacia su figura. Todos estos comportamientos pueden generar en el terapeuta sentimientos de enfado, frustración, impotencia y desgaste emocional, pudiendo llegar al burn-out (Taft y Murphy, 2007; Ross et al., 2008). La exposición del terapeuta en el grupo es permanente, lo que requiere un buen manejo de este tipo de sentimientos, esencial para el buen funcionamiento del grupo. Así, el terapeuta debe poseer una buena auto gestión y control emocional que le permita detectar y manejar estas emociones (Ross et al., 2008). Además, resultará también esencial que posea altos niveles de tolerancia a la frustración ante las resistencias del grupo y el fracaso de alguna de las intervenciones, ya que forman parte del proceso de tratamiento con esta población. Del mismo modo, realizar una buena gestión emocional puede verse favorecida por el apoyo de una figura de supervisión y disponer de apoyo institucional que permita un buen clima laboral (Carbajosa et al., 2013).

En definitiva, la intervención grupal en estos programas implica un enorme reto para el terapeuta. El trabajo con una población poco motivada y resistente al tratamiento o las demandas del contexto judicial hacen esta tarea compleja y convierten al terapeuta en un verdadero equilibrista.

\section{Referencias}

Ackerman, S. J. y Hilsenroth, M. J. (2003). A review of therapist characteristics and techniques positively impacting the therapeutic alliance. Clinical Psychology Review, 23, 1-33.

Agoff, C., Herrera, C. y Castro, R. (2007). The weakness of family ties and their perpetuating effects on gender violence: A qualitative study in Mexico. Violence Against Women, 13, 1206-1220.

Alexander, P. C., Morris, E., Tracy, A. y Frye, A. (2010). Stages of change and the group treatment of batterers: A randomized clinical trial. Violence and Victims, 25, 571-587.

Andrews, D. A., Bonta, J. y Wormith, J. S. (2011). The Risk-Need-Responsivity (RNR) model: Does adding the Good Lives Model contribute to effective crime prevention? Criminal Justice and Behavior, 38, 735755.

Arce, R. y Fariña, F. (2007). Intervención psicosocial con maltratadores de género. En J. M. Sabucedo y J. Sanmartín (Eds.), Los escenarios de la violencia (pp. 29-43). Barcelona: Ariel.

Boira, S. (2010). Hombres maltratadores. Historias de violencia masculina. Zaragoza: Prensas Universitarias de Zaragoza.

Boira, S., Del Castillo, M., Carbajosa, P. y Marcuello, Ch. (2013). Context of treatment and therapeutic alliance: Critical factors in the intervention with court-ordered batterers. The Spanish Journal of Psychology, 16 (40), $1-13$.

Boira, S., Lopez, Y., Tomas-Aragones, L. y Gaspar, A. R. (2013). Efficacy of different treatment modalities in men convicted of intimate partner violence. Anales de Psicología, 29, 19-28.

Bordín, E. S. (1979). The generalizability of the psychoanalytic concept of the working alliance. Psychotherapy Theory Research Practice, 16, 252-260.

Bouman, Y. H. A., Schene, A. H. y Ruiter, C. (2009). Subjective well-being and recidivism in forensic psychiatric outpatients. International Journal of Forensic Mental Health, 8, 225-234.

Bowen, E. (2011). The rehabilitation of partner-violent men. Oxford: Wiley-Blackwell.

Carbajosa, P. y Boira, S. (2013). Estado actual y retos futuros de los programas para hombres condenados por violencia de género en España. Psychosocial Intervention. 22, 145-152. 
Carbajosa, P., Boira, S. y Tomas-Aragonés, L. (2013). Difficulties, skills and therapy strategies in interventions with court-ordered batterers in Spain. Aggression and Violent Behavior, 18, 118-124.

Choi, S. Y. P., Cheung, Y. W. y Cheung, A. K. L. (2012). Social isolation and spousal violence: Comparing female marriage migrants with local women. Journal of Marriage and Family, 74, 444-461.

Cirillo, S. (1994). El cambio en los contextos no terapéuticos. Barcelona: Paidós.

De Lemus, S. y Ryan, E. (2010). Relaciones de género en la infancia y adolescencia: desarrollo de las actitudes sexistas. En E. Ryan y S. Lemus (Eds.), Coeducación: propuestas para alcanzar la igualdad de género desde las aulas (pp. 69-100). Granada: Universidad de Granada.

Diaz-Aguado, M. J. (2006). Sexismo, violencia de género y acoso escolar. Propuestas para una prevención integral de la violencia. Revista de Estudios de Juventud, 73, 38-57.

Dutton, D. y Golant, S. K. (1997). El golpeador. Un perfil psicológico. Buenos Aires: Paidós.

Echauri, J. A., Romero, J. y Rodríguez, M. J. (2005). Teoría y descripción de la violencia de género. Programa terapéutico para maltratadores del ámbito familiar en el centro penitenciario de Pamplona. Anuario de Psicología Jurídica, 15, 67-95.

Echeburúa, E. (2013). Adherencia al tratamiento en hombres maltratadores contra la pareja en un entorno comunitario: Realidad actual y retos de futuro. Psychosocial Intervention, 22, 87-93.

Echeburúa, E., Sarasua, B., Zubizarreta, I. y de Corral, P. (2009). Evaluación de la eficacia de un tratamiento cognitivo-conductual para hombres violentos contra la pareja en un marco comunitario: Una experiencia de 10 años (1997-2007). International Journal of Clinical and Health Psychology, 9, 199-217.

Eckhardt, C. I., Babcock, J. y Homack, S. (2004). Partner assaultive men and the stages and processes of change. Journal of Family Violence, 19, 81-93.

Eckhardt, C., Holtzworth-Munroe, A., Norlander, B., Sibley, A. y Cahill, M. (2008). Readiness to change, partner violence subtypes, and treatment outcomes among men in treatment for partner assault. Violence and Victims, 23, 446-475.

Eckhardt, C. I., Murphy, C. M., Whitaker, D. J., Sprunger, J., Dykstra, R. y Woodard, K. (2013). The effectiveness of intervention programs for perpetrators and victims of intimate partner violence. Partner Abuse, 4 , 196-231.

Eckhardt, C., Samper, R. y Murphy, C. (2008). Anger disturbance among perpetrators of intimate partner violence. Clinical characteristics and outcomes of court-mandated treatment. Journal of Interpersonal Violence, 23, 1600-1617.

Edleson, J. L. y Tolman, R. M. (1992). Ecological interventions for domestic violence. Thousand Oaks, CA: Sage.

Feder, L., Wilson, D. B. y Austin, S. (2008). Court-mandated interventions for individual convicted of domestic violence. Campbell Systematic Reviews, 12.

Gondolf, E. (2012). The future of batterer programs: Reassessing evidence-based practice. Boston: Northeastern University Press.

Gracia, E., García, F. y Lila, M. (2009). Public responses to intimate partner violence against women: The influence of perceived severity and personal responsibility. The Spanish Journal of Psychology, 12, 648656.

Gracia, E., Herrero, J., Lila, M. y Fuente, A. (2009). Perceived neighborhood social disorder and attitudes toward domestic violence against women among Latin-American immigrants. European Journal of Psychology Applied to Legal Context, 1, 25-43.

Graham, K., Bernards, S., Flynn, A., Tremblay, P. F. y Wells, S. (2012). Does the relationship between depression and intimate partner aggression vary by gender, victim-perpetrator role, and aggression severity? Violence and Victims, 27, 730-743.

Graña, J., Muñoz, M., Redondo, N. y González, M. (2008). Programa para el tratamiento psicológico de maltratadores. Madrid: Servicio de Publicaciones de la Universidad Complutense. 
Henning, K., Jones, A. R. y Holdford, R. (2005). “I didn’t do it, but if I did I had a good reason”: Minimization, denial, and attributions of blame among male and female domestic violence offenders. Journal of Family Violence, 20, 131-139.

Kistenmacher, B. R. y Weiss, R. L. (2008). Motivational interviewing as a mechanism for change in men who batter: A randomized control trial. Violence and Victims, 23, 558-570.

Kozar, C. J. y Day, A. (2012). The therapeutic alliance in offending behavior programs: A necessary and sufficient condition for change? Aggression and Violent Behavior,17, 482-487.

Langlands, R. L., Ward, T. y Gilchrist, E. (2009). Applying the Good Lives Model to male perpetrators of domestic violence. Behaviour Change, 26, 113-129.

Lanier, C. y Maume, M. O. (2009). Intimate partner violence and social isolation across the rural/urban divide. Violence Against Women, 15, 1311-1330.

Lee, M. Y., Sebold, J. y Uken, A. (2003). Solution-focused treatment with domestic violence offenders: Accountability for change. New York: Oxford University Press.

Lila, M. (2013). La intervención con hombres condenados por violencia de pareja contra la mujer en España: Investigación y avances en intervención. Psychosocial Intervention, 22, 81-85.

Lila, M., García, A. y Lorenzo, M. V. (2010). Manual de intervención con maltratadores. Valencia: Servicio Publicaciones Universidad de Valencia.

Lila, M., Gracia, E. y Herrero, J. (2012). Asunción de responsabilidad en hombres maltratadores: influencia de la autoestima, la personalidad narcisista y la personalidad antisocial. Revista Latinoamericana de Psicología, 44, 99-108.

Lila, M., Gracia, E. y Murgui, S. (2013). Psychological adjustment and victim-blaming among intimate partner violence offenders: The role of social support and stressful life events. European Journal of Psychology Applied to Legal Context, 5, 147-153.

Lila, M., Herrero, J. y Gracia, E. (2008). Evaluating attribution of responsibility and minimization by male batterers: Implications for batterer programs. The Open Criminology Journal, 1, 4-11.

Lila, M., Oliver, A., Galiana, L. y Gracia, E. (2013). Predicting success indicators of an intervention programme for convicted intimate-partner violence offenders: The Contexto Programme. European Journal of Psychology Applied to Legal Context, 5, 73-95.

Lila, M., Oliver, A., Catalá-Miñana, A., Galiana, L. y Gracia, E. (2014). The Intimate Partner Violence Responsibility Attribution Scale (IPVRAS). European Journal of Psychology Applied to Legal Context, 6, 29-36.

Lila, M., Oliver, A., Lorenzo, M. V. y Catalá, A. (2013). Valoración del riesgo de reincidencia en violencia contra la mujer en las relaciones de pareja: Importancia del apoyo social. Revista de Psicología Social, 28, 225236.

Marshall, W. L. (2005). Therapist style in sexual offender treatment: Influence on indices of change. Sexual Abuse: A Journal of Research and Treatment, 17, 109-116.

Marshall, W. L., Serran, G. A., Moulden, H., Mulloy, R., Fernandez, Y. M., Mann, R. E. y Thornton, D. (2002). Therapist features in sexual offender treatment: Their reliable identification and influence on behavior change. Clinical Psychology and Psychotherapy, 9, 395-405.

Millana, L. (2011). Intervention programs for Spanish inmate aggressors convicted of domestic violence. The Open Criminology Journal, 4, 91-101.

Miller, W. R. y Rollnick, S. (2002). Motivational interviewing: Preparing people for change (2nd ed.). New York: Guilford.

Murphy, C. M. y Baxter, V. A. (1997). Motivating batterers to change in the treatment context. Journal of Interpersonal Violence, 12, 607-619.

Murphy, C. M. y Eckhardt, C. I. (2005). Treating the abusive partner: An individualized cognitive-behavioral approach. New York: The Guilford Press. 
Murphy, C. M., Linehan, E. L., Reyner, J. C., Musser, P. H. y Taft, C. T. (2012). Moderators of response to motivational interviewing for partner-violent men. Journal of Family Violence, 27, 671-680.

Murphy, C. M., Stosny, S. y Morrel, T. M. (2005). Change in self-esteem and physical aggression during treatment for partner violent men. Journal of Family Violence, 20, 201-210.

Novo, M., Fariña, F., Seijo, D. y Arce, R. (2012). Assessment of a community rehabilitation programme in convicted male intimate-partner violence offenders. International Journal of Clinical and Health Psychology, 12, 219-234.

Organización Mundial de la Salud (2013). Global and regional estimates of violence against women: Prevalence and health effects of intimate partner violence and non-partner sexual violence. Ginebra, Suiza: Organización Mundial de la Salud.

Pérez, M., Giménez-Salinas, A. y de Juan, M. (2013). Evaluación de la eficacia del programa de tratamiento con agresores de pareja (PRIA) en la comunidad. Psychosocial Intervention, 22, 105-114.

Polaschek, D. L. y Ross, E. (2010). Do early therapeutic alliance, motivation and stages of change predict therapy change for high-risk psychopathic violent prisoners. Criminal Behavior and Mental Health, 20, 100-111.

Price, B. J. y Rosembaum, A. (2009). Batterer intervention programs: A report from the field. Violence and Victims, 24, 757-770.

Prochaska, J. y DiClemente, C. (1986). The Transtheoretical approach. En J. Norcross (Eds.), Handbock of eclectic psychoterapy (pp. 163-200). New York: Brumer/Mazel.

Quinteros, A. y Carbajosa, P. (2008). Hombres maltratadores: tratamiento psicológico de agresores. Madrid: Grupo 5 Acción y Gestión Social.

Rasanen, E., Holma, J. y Seikkula, J. (2012). Dialogical views on partner abuser treatment: Balancing confrontation and support Journal of Family Violence, 27, 357-368.

Redondo, S. (2010). Manual para el tratamiento psicológico de los delincuentes. Madrid: Pirámide.

Redondo, S., Martínez-Catena, A. y Andrés-Pueyo, A. (2012). Efectos terapéuticos de un tratamiento cognitivoconductual con delincuentes juveniles. European Journal of Psychology Applied to Legal Context, 4, 159-178.

Rodriguez, L., Antuña, M. A., López-Cepero, J., Rodríguez. F. J., Herrero, F. J. y Bringas. C. (2008). Una aproximación al estudio de las actitudes sexistas en novios adolescentes. En F. J. Rodríguez, C. Bringas, F. Fariña, R. Arce y A. Bernardo (Eds.), Psicología jurídica: familia y victimología (pp. 219-227). Oviedo: Sociedad Española de Psicología Jurídica y Forense.

Romero-Martínez, A., Lila, M., Catalá-Miñana, A., Williams, R. K. y Moya-Albiol, L. (2013). The contribution of childhood parental rejection and early androgen exposure to impairments in socio-cognitive skills in intimate partner violence perpetrators with high alcohol consumption. International Journal of Environmental Research and Public Health, 10, 3753-3770.

Rosenberg, M. (2003). Voices from the group: Domestic violence offenders' experience of intervention. Journal of Aggression, Maltreatment, \& Trauma, 7, 305-317.

Ross, E., Polaschek, D. y Ward, T. (2008). The therapeutic alliance: A theoretical revision for offender rehabilitation. Aggression and Violent Behavior, 13, 462-480.

Ruiz, S. y Expósito, F. (2008). Intervención específica con internos condenados por delito de violencia de género. Colección Psicología y Ley, 5, 389-397.

Saunders, D. G. (2008). Group interventions for men who batter: A summary of program descriptions and research. Violence and Victims, 23, 156-172.

Scott, K. L. (2004). Stage of change as a predictor of attrition among men in a batterer treatment program. Journal of Family Violence, 19, 37-47.

Scott, K. L., King, C., McGinn, H. y Hosseini, N. (2011). Effects of motivational enhancement on immediate outcomes of batterer intervention. Journal of Family Violence, 26, 139-149.

Scott, K. L. y Wolfe, D. A. (2003). Readiness to change as a predictor of outcome in batterer treatment. Journal of Consulting and Clinical Psychology, 71, 879-889. 
Secretaría General de Instituciones Penitenciarias (2011). Informe General 2010. Madrid: Ministerio del Interior. Recuperado de http://www.institucionpenitenciaria.es/web/portal/documentos/publicaciones.html

Silver, E. y Teasdale, B. (2005). Mental disorder and violence: An examination of stressful life events and impaired social support. Social Problems, 52, 62-78.

Silvergleid, C. S. y Mankowski, E. S. (2006). How batterer intervention programs work: Participant and facilitator accounts of processes of change. Journal of Interpersonal Violence, 21,139-159.

Sonkin, D. J. y Dutton, D. G. (2003). Treating assaultive men from an attachment perspective. Journal of Aggression, Maltreatment, and Trauma, 7, 105-133.

Stewart, L. A., Flight, J. y Slavin-Stewart, C. (2013). Applying effective corrections principles (RNR) to partner abuse interventions. Partner Abuse, 4, 494-534.

Subirana-Malaret, M. y Andrés-Pueyo, A. (2013). Retención proactiva y adherencia terapéutica en programas formativos para hombres maltratadores de la pareja. Psychosocial Intervention, 22, 95-104.

Taft, C. T. y Murphy, C. M. (2007). The working alliance in intervention for partner violence perpetrators: Recent research and theory. Journal of Family Violence, 22, 11-18.

Taft, C. T., Murphy, C. M., Musser, P. H. y Remington, N. A. (2004). Personality, interpersonal, and motivational predictors of the working alliance in group cognitive-behavioral therapy for partner violent men. Journal of Consulting and Clinical Psychology, 72, 349-354.

Taft, C. T., Murphy, C. M., King, D. W., Musser, P. H. y DeDeyn, J. M. (2003). Process and treatment adherence factors in group cognitive-behavioral therapy for partner violent men. Journal of Consulting and Clinical Psychology, 71, 812-820.

Zalmanowitz, S., Babins-Wagner, R., Rodger, S., Corbett, B. A. y Leschied, A. (2013). The association of readiness to change and motivational interviewing with treatment outcomes in males involved in domestic violence group therapy. Journal of Interpersonal Violence, 28, 956-974.

Manuscrito recibido: 20/01/2014

Revisión recibida: 13/02/2014

Manuscrito aceptado: 19/02/2014 